\title{
Brain-derived neurotrophic factor Val66Met and blood glucose: a synergistic effect on memory
}

\author{
Naftali Raz ${ }^{1,2, *}$, Cheryl L. Dahle ${ }^{1,2}$, Karen M. Rodrigue ${ }^{2}$, Kristen M. Kennedy², \\ Susan J. Land ${ }^{3}$ and Bradley S. Jacobs ${ }^{4}$
}

1. Department of Psychology, Wayne State University, Detroit, MI, USA

2. Institute of Gerontology, Wayne State University, Detroit, MI, USA

3. Department of Obstetrics and Gynecology, Wayne State University, Detroit, MI, USA

4. Department of Internal Medicine, Wright State University, Dayton, OH, USA

Edited by: William J. Jagust, University of California Berkeley, USA

Reviewed by: Bradford C. Dickerson, Harvard Medical School, USA

William J. Jagust, University of California Berkeley, USA

\begin{abstract}
Age-related declines in episodic memory performance are frequently reported, but their mechanisms remain poorly understood. Although several genetic variants and vascular risk factors have been linked to mnemonic performance in general and age differences therein, it is unknown whether and how they modify age-related memory declines. To address that question, we investigated the effect of Brain-Derived Neurotrophic Factor (BDNF) Val66Met polymorphism that affects secretion of BDNF, and fasting blood glucose level (a vascular risk factor) on episodic memory in a sample of healthy volunteers (age 19-77). We found that advanced age and high-normal blood glucose levels were associated with reduced recognition memory for name-face associations and poorer prose recall. However, elevated blood glucose predicted lower memory scores only in carriers of the BDNF 66Met allele. The effect on associative memory was stronger than on free recall. These findings indicate that even low-level vascular risk can produce negative cognitive effects in genetically susceptible individuals. Alleviation of treatable vascular risks in such persons may have a positive effect on age-related cognitive declines.
\end{abstract}

Keywords: aging, BDNF, memory, vascular risk, single nucleotide polymorphism, paired-associates, recognition, free recall

\section{INTRODUCTION}

Episodic memory declines with age (Verhaeghen et al., 1993) and associative memory tasks that require binding of multiple pieces of information may be especially sensitive to aging (Old and Naveh-Benjamin, 2008). However, biological underpinnings of age-related memory declines remain unclear. Multiple factors, including heredity (Finkel et al., 1995) and metabolic characteristics (e.g., glycemic control; Rolandsson et al., 2008; Vanhanen et al., 1997; Yaffe et al., 2004) affect memory performance, but little is known about whether and how interactions among them contribute to age-related differences in memory.

Recent developments in genomic technology have advanced a more nuanced understanding of how specific genetic variations influence cognitive skills. Among single-nucleotide polymorphisms (SNPs) that affect cognitive performance, some have been linked specifically to episodic memory. One such SNP is a variant in a gene that controls secretion of the Brain-Derived Neurotrophic Factor (BDNF) in the central nervous system

*Correspondence: Naftali Raz, Institute of Gerontology, 87 E Ferry St., 226 Knapp, Detroit, MI 48202, USA. e-mail: nraz@wayne.edu

Received: 04 August 2008; paper pending published: 31 August 2008; accepted: 18 September 2008; published online: 03 0ctober 2008.

Citation: Front. Hum. Neurosci. (2008) 2: 12. doi: 10.3389/neuro.09.012.2008

Copyright $\odot 2008$ Raz, Dahle, Rodrigue, Kennedy, Land and Jacobs. This is an openaccess article subject to an exclusive license agreement between the authors and the Frontiers Research Foundation, which permits unrestricted use, distribution, and reproduction in any medium, provided the original authors and source are credited.
(CNS). BDNF plays an important role in cellular mechanisms of memory consolidation: calcium-dependent modifications of dendritic structure, synaptogenesis, and long-term potentiation (Finkbeiner, 2000; Lynch et al., 2006). The low-activity (Met) allele of a common BDNF polymorphism (Val66Met) is associated with reduced BDNF expression (Egan et al., 2003), and may confer disadvantage in performance on memory tasks (Bath and Lee, 2006). To date, six studies in non-pathological populations found that carriers of the BDNF Met66 allele have poorer verbal memory than Val homozygotes (Dempster et al., 2005; Egan et al., 2003; Goldberg et al., 2008; Ho et al., 2006; Miyajima et al., 2007; Raz et al., in press) and one study of younger adults found a similar Val/Val advantage in a scene recognition task (Hariri et al., 2003). In contrast, two studies revealed no association between BDNF Val66Met and memory (Hansell et al., 2007; Harris et al., 2006). Thus the preponderance of evidence reported to date indicates that the BDNF 66Met allele may be a negative modifier of memory.

Aging is associated with a reduction of BDNF expression during learning-related plasticity (Sohrabji and Lewis, 2006), and therefore factors that affect BDNF secretion may explain some of the age-related variability in memory. Little is known about the effect of BDNF on age differences in memory, as the vast majority of the extant studies have not employed samples covering a sufficiently broad age range. Furthermore, most of the available studies of BDNF Val66Met and memory did not take into account physiological characteristics and environmental 
factors that may influence the expression of genes and thus affect cognitive performance. Physiological risk factors may interact with genetic risk to exert an adverse effect on cognitive performance. Indeed, we found that hypertension has a negative effect on speed of processing in carriers of the BDNF 66Met allele (Raz et al., in press). Although we found a significant negative effect of BDNF 66Met allele on memory in that study, no interactions with hypertension were observed. Nonetheless, it is possible that other indicators of vascular risk and vascular health more closely associated with BDNF activity are capable of affecting memory.

One group of factors that may act as age-dependent negative modifiers of cognitive performance consists of variables associated with glycemic control. High levels of blood glucose that contribute to cardiovascular morbidity (Coutinho et al., 1999) have a negative impact on memory in older adults, even at elevations short of a clinical diagnosis of diabetes (Riby et al., 2008; Rolandsson et al., 2008). Notably, BDNF and glucose are mutually dependent factors in multiple neural processes. BDNF facilitates glucose uptake in the brain (Mattson et al., 2004), increase in blood glucose levels inhibits brain release of BDNF (Krabbe et al., 2007), BDNF-deficient knockout mice develop hyperglycemia (Lyons et al., 1999), and BDNF administration improves glucose metabolism in experimental diabetes (Nakagawa et al., 2002). Thus, genetic reduction in BDNF release may affect cognition in a specific biochemical environment, such as elevated glucose levels, and genetic and metabolic factors may synergistically influence memory. Moreover, such synergistic effects may be exacerbated by old age. Furthermore, in persons with genetic predisposition, lesser dose of a potential risk factor such as highnormal blood glucose may be needed to produce the effect.

Therefore, in this study we examined the joint effects of BDNF Val66Met genotype and blood glucose on episodic memory in a healthy adult lifespan sample. We hypothesized that BDNF Val66 homozygotes would perform better than BDNF Met66 carriers on an associative memory task, with lesser or no effect of the genotype expected on a task with no associative (binding) component. Furthermore, we hypothesized that higher blood glucose would be associated with poorer memory performance and the difference would be exacerbated by lower CNS levels of BDNF as expected for the low-activity allele of the Val66Met polymorphism. To test the hypothesis that higher, but clinically normal, blood glucose levels would be associated with reduced memory performance in genetically susceptible individuals, we selected a sample of normotensive and normoglycemic adults.

\section{MATERIALS AND METHODS \\ PARTICIPANTS}

The participants were recruited as a part of an ongoing longitudinal study of healthy aging through local media. They were screened via health questionnaire and a telephone interview for history of cardiovascular, neurological and psychiatric conditions, head trauma, alcohol and drug abuse, thyroid problems, and diabetes. The participants had corrected visual acuity of 50/20 or better (Optic 2000, Stereo Optic) and hearing of $40 \mathrm{~dB}$ or better for frequencies of $500-4000 \mathrm{~Hz}$ (Maico, MA27). They were screened for dementia and depression with the Mini-Mental State Examination (MMSE; Folstein et al., 1975) and the Geriatric Depression Questionnaire (CES-D; Radloff, 1977). Only persons who scored 26 or above on MMSE and 15 or below on CES-D were invited to participate. All participants provided written informed consent in accord with the Wayne State University Human Investigation Committee.

The full complement of genetic, blood, and memory data were available for 103 volunteers (73 women, age range 19-77, mean \pm SD: $53.24 \pm 14.23$ ), who were part of a larger sample of 189 participants ( $\mathrm{Raz}$ et al., in press). The participants were normotensive (systolic blood pressure 91-140 mm Hg, $118.58 \pm 10.90 \mathrm{~mm} \mathrm{Hg}$; diastolic blood pressure 60-89 $\mathrm{mm} \mathrm{Hg}$, $73.50 \pm 6.63 \mathrm{~mm} \mathrm{Hg}$ ), and normoglycemic (fasting blood glucose $86.59 \pm 9.32,66-113 \mathrm{mg} / \mathrm{dl})$. The education was $15.75 \pm$ 2.06 years. MMSE scores ranged from 26 to 30 (29.10 \pm 0.92$)$. Men and women did not differ in age $(p=0.67)$, MMSE $(p=0.26)$, or systolic blood pressure $(p=0.23)$. There was a trend for men to have slightly higher glucose levels $(p=0.10)$ and higher diastolic blood pressure $(p=0.07)$ than women. Men had less than a year more schooling than women $(p<0.05)$. Only $8 \%$ of the participants smoked (21-27\% expected in the general population) and $81 \%$ reported exercising regularly $(31 \%$ expected in the general population) (American Heart Association, 2005). Thus, the participants were apparently healthier and better educated than a typical sample from the general population.

\section{MEASURES OF VASCULAR HEALTH INDICATORS}

Following a 12-h overnight fast whole blood glucose levels were assessed by standard enzymatic glucose oxidase method, and persons with blood glucose levels below $126 \mathrm{mg} / \mathrm{dl}(<7.0 \mathrm{mmol} / \mathrm{l})$ were retained. Blood pressure was measured from the left arm of seated participants by a mercury sphygmomanometer (BMS 12-S25) with a standard cuff (Omron Professional) on three separate days before administration of the cognitive tasks. Persons with mean systolic pressure of $140 \mathrm{~mm} \mathrm{Hg}$ or mean diastolic pressure of $90 \mathrm{~mm} \mathrm{Hg}$ and above were excluded.

\section{GENOMIC ANALYSIS}

DNA was isolated from buccal cultures in mouthwash samples using a Gentra Autopure LS under the standard buccal cell protocol. For quality control, 10\% direct repeats and DNA sequencing for verification were performed with control DNA and no-template controls. All 5' -nuclease assays were run on an Applied Biosystems 7900. Polymorphism for BDNF (rs6265) was interrogated using a Taqman SNP Genotyping assay. DNA sequencing reactions were carried out using the $0.5 \times$ protocol for ABI PRISM BigDye Terminator Cycle Sequencing Ready Reaction Kit (Applied Biosystems). The sequencing extension products were purified utilizing Sephadex, and analyzed on an ABI PRISM 3700 DNA Analyzer using a $50 \mathrm{~cm}$ capillary array.

The distribution of the BDNF Val66Met alleles fit the Hardy-Weinberg equilibrium $\left(\chi^{2}=0.13\right.$, ns $)$, with $60 \%$ being Val homozygotes, $34 \% \mathrm{Val} / \mathrm{Met}$ heterozygotes, and $6 \%$ with the Met/Met genotype. Due to the rarity of Met/Met in Caucasians in this sample and in the general population (http://www.ncbi. nlm.nih.gov/SNP/snp_ref.cgi?rs=6265, accessed July 26, 2008), the alleles were grouped as BDNF Met+ $(n=41)$ and BDNF Met- (i.e., Val/Val, $n=62$ ). Met carriers and Val/Val homozygotes did not differ in age: $t(101)=0.58$, ns.

\section{MEMORY MEASURES}

Name-picture associative memory.

Memory for Names from the Woodcock-Johnson Psychoeducational Battery-Revised (WJPB-R; Woodcock and Johnson, 1989) measured associative memory. In the task, participants 
viewed 12 consecutive line drawings of novel stimuli (cartoons of "space creatures") and were told each creature's "name", a nonsense word. During study, participants viewed 12 plates depicting space creatures, one "new" creature and eight foils per plate. The task was to point to the creature named by the examiner. After a 20-min delay, the creatures (target plus eight alternatives per plate) were displayed again and the participant was asked to point to the creature named by the experimenter. At the delayed administration, each plate was displayed three times for a total of 36 trials. The performance indices were the total number of correct matches for immediate and delayed recognition. The reliability of the immediate and delayed scores is 0.91 (Woodcock and Mather, 1989).

\section{Story recall}

Recall of a prose passage was assessed by the Logical Memory (LM) subtest from the Wechsler Memory Scale-Revised (WMS-R; Wechsler, 1987). Participants heard two short stories of just a few lines each. Following each presentation they were asked to relay the story verbatim. After a 20-min delay, participants were again asked to repeat the stories as close to the original wording as they remembered. If they were unable to do so, a simple reminder cue was provided for one or both stories. The split-half reliability of this task is estimated at 0.74 for immediate and 0.75 for delayed presentation (Wechsler, 1987).

\section{STATISTICAL ANALYSES}

The data for each test were analyzed in two separate General Linear models. Memory performance indices (number of correct associations or number of correct prose segments) were dependent variables. Study-test delay was a repeated measures factor. Age and blood glucose levels, centered at sample means, were continuous predictors; sex and BDNF genotype (Met carriers vs. Val homozygotes) were categorical predictors. Second order interactions among all predictors were included in the models, but deleted if found nonsignificant $(p>0.10)$; the reduced models were then evaluated. We tested no higher-order interactions because some categories created by crossing of factors contained too few cases.

\section{RESULTS}

The correlations among age, blood glucose levels, and episodic memory scores are presented in Table 1. Fasting blood glucose levels were moderately related to age, but not to BDNF genotype: $t(101)=0.33$, ns.

\section{MEMORY FOR NAME-PICTURE ASSOCIATIONS}

Results of the general linear model analysis are summarized in Table 2. The main effects of age, blood glucose level, and

Table 1 | Memory, age, and fasting blood glucose: zero-order correlations.

\begin{tabular}{llllll}
\hline & Age & Glucose & LM & LMd & NP \\
\hline Blood glucose & $0.36^{\ddagger}$ & & & & \\
Logical Memory & -0.20 & -0.13 & & & \\
Logical Memory, delayed & $-0.22^{*}$ & -0.13 & $0.89^{\ddagger}$ & & \\
Name-picture & $-0.48^{\ddagger}$ & $-0.25^{*}$ & $0.35^{\ddagger}$ & $0.39^{\ddagger}$ & \\
Name-picture, delayed & $-0.44^{\ddagger}$ & $-0.33^{\dagger}$ & $0.44^{\ddagger}$ & $0.47^{\ddagger}$ & $0.87^{\ddagger}$ \\
\hline
\end{tabular}

$* p<0.05,{ }^{\dagger} p<0.01,{ }^{\ddagger} p<0.001$.

LM, Logical Memory; LMd, Logical Memory, delayed recall; NP, name-picture. study-test delay were significant. Poorer memory for namepicture associations was linked with older age, higher blood glucose levels, and a delay between study and test. The effect of blood glucose on association memory was modified by significant interactions: $\mathrm{BDNF} \times$ Glucose and Delay $\times \mathrm{BDNF} \times$ Glucose. The interactions reflected the impact of the BDNF genotype on strength of association between glucose levels and memory. Significant correlations between memory and blood glucose levels were observed only among the BDNF 66Met carriers: $r=-0.50$ for immediate and $r=-0.49$ for delayed recognition, both $p<0.001$. As is shown in Figure 1, no association between blood glucose and immediate memory score was noted for Val homozygotes: $r=0.14$, ns; a trend for delayed recognition was observed: $r=-0.25, p<0.06$.

Although the main effect of age was statistically modified by BDNF genotype and a delay between study and test, decomposition of that interaction revealed only small and probably trivial differences. Specifically, among Val homozygotes, age differences were stronger in immediate than delayed recall $(r=-0.51$, $p<0.001$ vs. $r=-0.42, p<0.01)$, and in the opposite direction for the Met carriers: $r=-0.44, p<0.001$, for immediate vs. $r=-0.50, p<0.001$ for delayed.

\section{STORY RECALL}

The main effect of age reflected better performance by younger participants. The magnitude of age differences in story recall was smaller than in associative memory: $Z^{*}=2.32, p<0.05$ for delayed, and $Z^{*}=2.67, p<0.01$ for immediate recall. The relation between blood glucose levels and delayed story recall was also weaker than for delayed associative memory: $Z^{*}=2.03$, $p<0.05$. The difference for immediate memory was not significant, albeit in the same direction: $Z^{*}=1.16$, ns. The modifying influence of BDNF Val66Met can be seen in Figure 2. Among BDNF Met carriers, higher glucose levels were associated with lower LM scores: $r=-.36, p<0.05$ for delayed recall and a trend $r=-.27, p<0.09$ for immediate recall. For BDNF Val homozygotes, no such associations were observed: $r=-.06$ for immediate and $r=0.00$ for delayed scores.

Table 2 | Effects of age, fasting blood glucose, and BDNF Val66Met polymorphism on episodic memory.

\begin{tabular}{lcc}
\hline \multicolumn{3}{c}{ Name-picture associations recognition } \\
\hline Effect & $\boldsymbol{F ( 1 , 9 6 )}$ & $\boldsymbol{p}$ \\
\hline Age & 20.79 & 0.001 \\
Glucose & 4.71 & 0.032 \\
Sex & 0.52 & 0.472 \\
BDNF & 1.43 & 0.235 \\
BDNF $\times$ glucose & 6.47 & 0.013 \\
Delay & 2546.34 & 0.001 \\
Delay $\times$ BDNF $\times$ age & 4.14 & 0.045 \\
Delay $\times$ BDNF $\times$ glucose & 4.25 & 0.042 \\
& Story recall & \\
Age & 4.60 & 0.035 \\
Delay & 81.85 & 0.001 \\
Delay $\times$ sex $\times$ glucose & 2.79 & 0.098 \\
Delay $\times$ glucose $\times$ BDNF & 3.95 & 0.050 \\
\hline
\end{tabular}

Nonsignificant main effects $(F<1)$ were included in the models but are not shown; nonsignificant interactions $(p>0.10$ ) were removed from the models. BDNF, Brain-derived neurotrophic factor; delay, immediate test versus test 20-min after study. 
A Immediate Name-Picture Association Recognition and Blood Glucose Modified by BDNF Val66Met

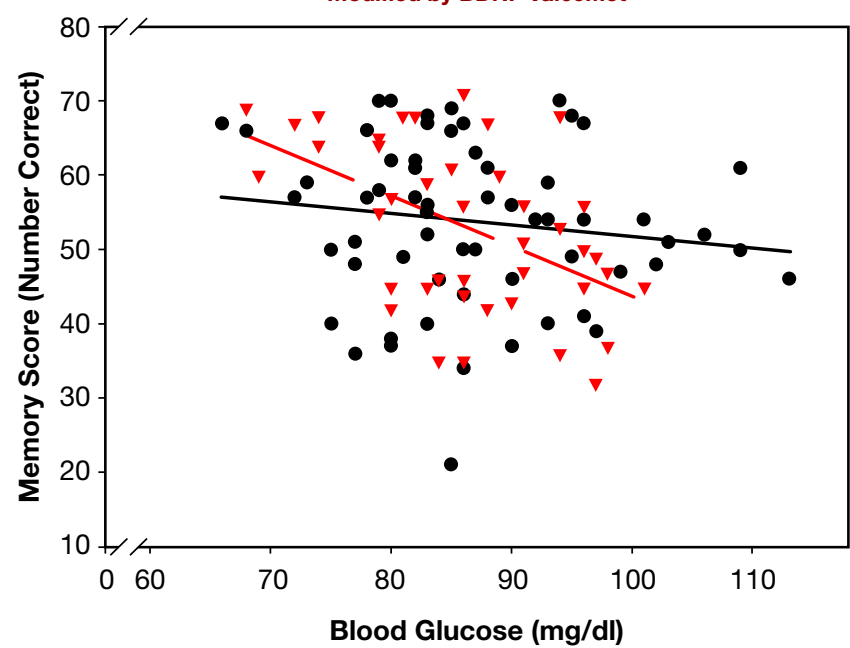
Modified by BDNF Val66Met

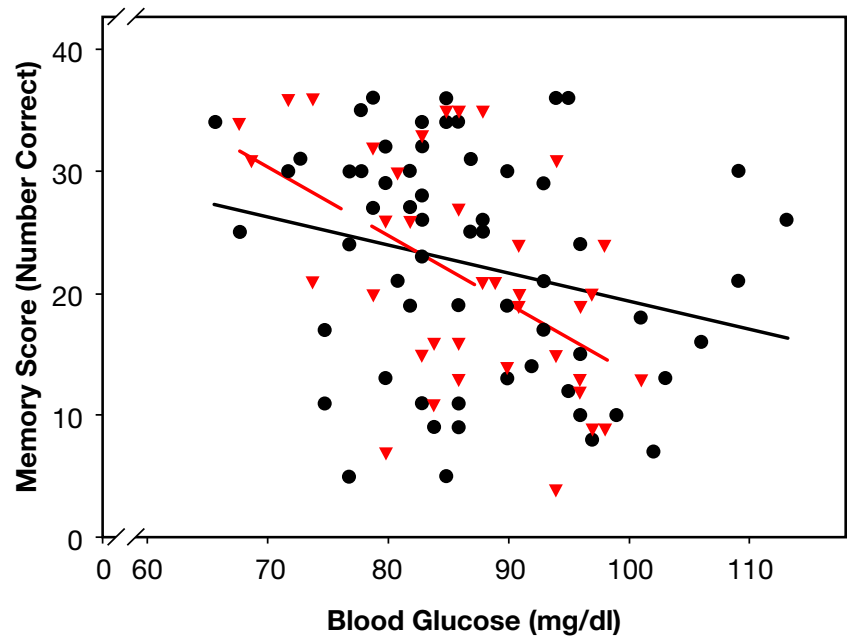

Figure 1 | Name-picture association recognition and blood glucose modified by BDNF Val66Met genotype. BDNF Val66Met Met carriers (red triangles, broken regression line) are compared to BDNF Val homozygotes (black circles, solid regression line). (A) Immediate recognition. (B) Delayed recognition.
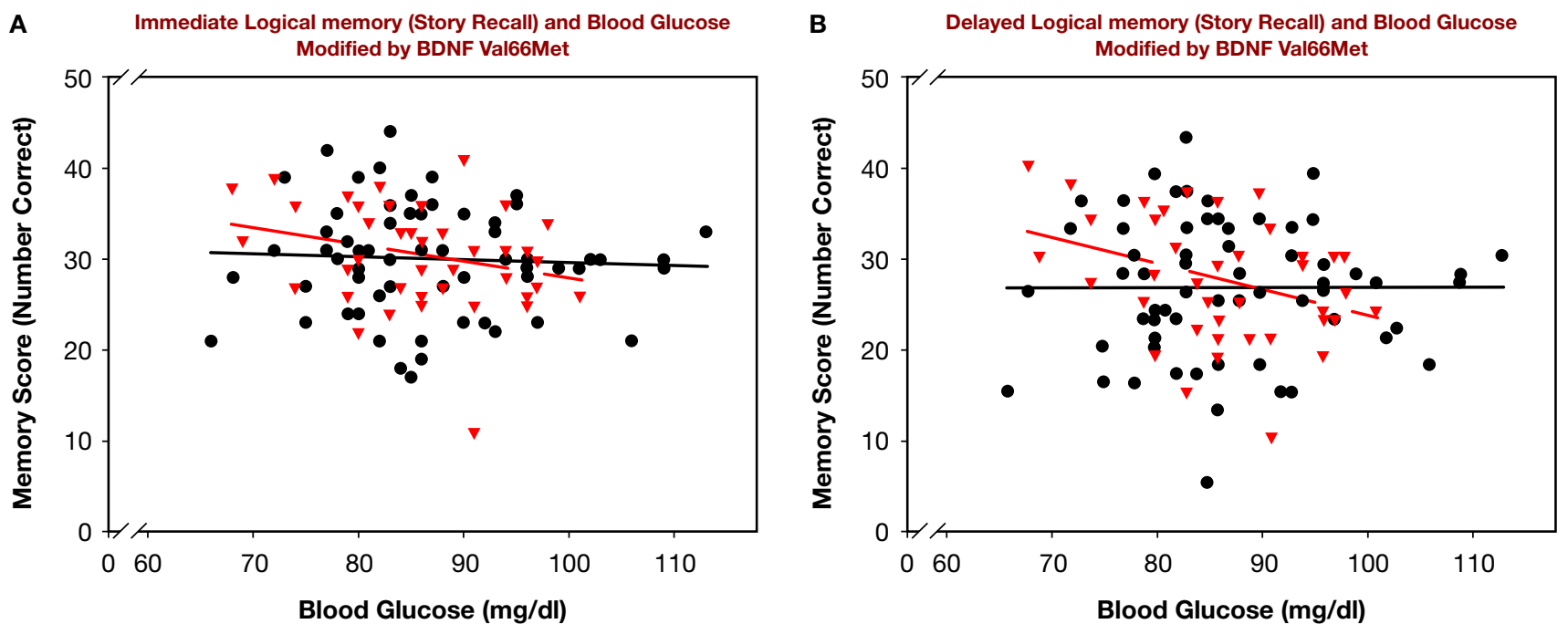

Figure 2 | Logical Memory (story recall) and blood glucose modified by BDNF Val66Met genotype. BDNF Val66Met Met carriers (triangles, broken regression line) are compared to BDNF Val homozygotes (circles, solid regression line). (A) Immediate recall. (B) Delayed recall.

\section{DISCUSSION}

As hypothesized, we found that in addition to age, high-normal fasting blood glucose has a negative impact on episodic memory, but this relationship was limited to carriers of the low-activity BDNF allele. Moreover, the synergistic effect of BDNF Met and blood glucose on memory was more pronounced in associative memory than prose recall. Whereas the age differences in memory and the link between memory and a vascular health indicator replicate results reported in the literature, modification of the glucose effect on memory by a genetic factor is a novel finding. Unlike our previous findings of BDNF Met effect on memory in a sample that contained persons with hypertension (Raz et al., in press), the reported synergistic effect was observed in healthy participants. Although truncation of glucose range probably produced a smaller effect than could have been observed in the full range of that variable, it is important to underscore that our findings were observed in a sample of persons unaffected by multiple health complications associated with clinically diagnosed diabetes mellitus.

Blood glucose level is a metabolic index statistically distributed within a rather wide range. Elevation of blood levels of glucose above an established cut-off, in conjunction with clinical symptoms, entails a diagnosis of diabetes. However, high normal blood glucose levels below the currently accepted cutoff for diabetes may represent early, preclinical diabetes. It is possible that individual cut-off values for a "normal" glucose level should be adjusted upwards or downwards depending on the genetic factors that affect glucose metabolism. Although largerscale studies are needed to assess the need for such adjustment, our results suggest that levels of glucose at the upper end of the clinically normal range may be associated with negative effects on memory in genetically predisposed individuals. A possibility 
that differential survival of Val homozygotes biased the findings was not a threat to validity in this sample as there were no age differences between the genotype groups.

One of the implications of our findings is that in comparison to carriers of the BDNF 66Met allele, Val homozygotes may be able to afford higher levels of glucose without detriment to their memory performance. The mechanisms underpinning the observed synergistic effect remain to be elucidated. Although increase in glucose levels inhibits BDNF release in the brain (Krabbe et al., 2007), it is unknown if chronic reduction in BDNF elevates glucose levels. In this sample, BDNF Met carriers did not have higher glucose levels than Val homozygotes. Thus, it is unlikely that genetically reduced BDNF resulted in higher blood glucose levels, and that elevations in glucose levels per se are responsible for memory deficits. The observed association is rather a marker of vulnerability in a genetically distinct group.

Glucose is not necessarily detrimental to memory performance. Moreover, acute glucose administration improves memory, especially in older adults (Gold, 2005; Messier, 2004), although the specificity of that effect has been challenged (Kaplan et al., 2000, 2001). Furthermore, memory performance may be negatively affected by glycemic dysregulation even at normal fasting glucose levels (Awad et al., 2002). Therefore, blood glucose level may be important for memory function inasmuch as it reflects other cellular and systemic processes. In healthy adults, elevated fasting glucose levels may reflect reduced insulin availability or insulin insensitivity, which suppresses memory scores in an age-dependent manner (Craft, 2005). Thus, additional research employing glucose regulation measures (i.e., insulin levels) may clarify the nature of BDNF modification of the link between memory and glucose metabolism. Because insulin levels were not assessed in the current study, this question remains open. It is also possible that increases in peripheral glucose levels do not imply its elevation in the brain. Brain and peripheral glucose levels are not controlled by the same mechanisms (Béquet et al., 2000) and chronic excess of extracellular glucose may be related to its reduced availability for intracellular processes, especially in the brain. Thus, our findings do not contradict the literature that links acute increases in blood glucose to improved memory. However, the effect of BDNF on such interventions is unknown.

The findings should be viewed in light of this study's limitations. First, although the BDNF-glucose interaction suggests a gene-environment interaction and possibly an epigenetic effect, it may in part be due to an interaction among multiple genes. It is unclear how much of the observed variance in fasting blood glucose is due to environment vs. genes. Investigations reported after completion of this study found that fasting blood glucose levels may be controlled by variants of the G6PC2 (BouatiaNaji et al., 2008) and ENPP1 (Stolerman et al., 2008) genes. The influence of those genes on memory functions sensitive to glucose levels merits future inquiry.

Second, although two memory indices were used in this study, not all components of episodic memory processes (e.g., encoding, consolidation, and retrieval) were assessed. These processes must be examined in greater detail to clarify the cognitive locus of the observed effect. A study focusing on the costs of associative binding is needed in order to establish whether specifically the associative component of memory is indeed more vulnerable to BDNF-glucose influence than other operations.

Third, one test measured recognition, whereas the other measured free recall. Because free recall is generally more sensitive to age effects and considered to be more difficult than recognition (Craik and McDowd, 1987), the results may reflect task difficulty. However, because the bias would be in the opposite direction than was found in this study, we have more confidence that the findings reflect the difference in memory modes. A tempting interpretation that greater effects of BDNF and blood glucose on associative memory indicate a BDNF role in a specific binding deficit should be viewed with caution. The prose paragraph recall task, though widely used in previous studies with mixed results (Dempster et al., 2005; Egan et al., 2003; Harris et al., 2006), is less reliable than the name-picture association task employed in this study. Thus, the difference in effect size may reflect differential reliability of the tests. Future investigations are needed to disentangle various cognitive mechanisms that may underlie the observed gene-vascular risk interaction on episodic memory.

Fourth, although the study sample afforded sufficient power to discover the interactive effects reported here, it was not sufficiently large to allow examination of higher-order interactions with other genes and other vascular risk factors. To establish if such interactions are possible, a much larger sample is needed.

Fifth, the cross-sectional design of the study does not permit assessment of true change, variability of change, or the establishment of causal links between glucose levels and cognitive performance. It is possible that high-normal glucose values observed in this sample are indicators of a pathological process that may produce higher and clinically significant values in a span of several years. Thus, the observed relationship may be a harbinger of an incipient metabolic disease. We hope that the longitudinal follow-up on participants of this study will shed light on the associations between age-related change in memory, genetic predisposition, and glycemic control.

In sum, we have demonstrated that genetic predisposition for reduced production of BDNF is associated with increased vulnerability to the negative effects of high-normal blood glucose levels in healthy adults without known vascular or metabolic disease. This synergistic effect may be more pronounced for memory tasks that require binding information and formation of associations. The results of this study suggest that interactions of specific genetic vulnerability and treatable vascular risks should be taken into account in conducting memory assessment and designing memory rehabilitation interventions for older adults.

\section{CONFLICT OF INTEREST STATEMENT}

The research was conducted in the absence of any commercial or financial relationships that could be construed as a potential conflict of interest.

\section{ACKNOWLEDGEMENTS}

This study was supported in part by grants R37-AG-11230 and T32 HS-013819 from the National Institutes of Health.

\section{REFERENCES}

American Heart Association. (2005). Heart Disease and Stroke Statistics: 2005 Update. Dallas, American Heart Association.

Awad, N., Gagnon, M., Desrochers, A., Tsiakas, M., and Messier, C. (2002). Impact of peripheral glucoregulation on memory. Behav. Neurosci. 116, 691-702.

Bath, K. G., and Lee, F. S. (2006). Variant BDNF (Val66Met) impact on brain structure and function. Cogn. Affect Behav. Neurosci. 6, 79-85.

Béquet, F., Pérès, M., Gomez-Mérino, D., Berthelot, M., Satabin, P., Piérard, C., and Guezennec, C. Y. (2000). Simultaneous NMR microdialysis study of brain 
glucose metabolism in relation to fasting or exercise in the rat. J. Appl. Physiol. 88, 1949-1954.

Bouatia-Naji, N., Rocheleau, G., Van Lommel, L., Lemaire, K., Schuit, F., Cavalcanti-Proença, C., Marchand, M., Hartikainen, A. L., Sovio, U., De Graeve, F., Rung, J., Vaxillaire, M., Tichet, J., Marre, M., Balkau, B., Weill, J., Elliott, P., Jarvelin, M. R., Meyre, D., Polychronakos, C., Dina, C., Sladek, R. and Froguel, P. (2008). A polymorphism within the G6PC2 gene is associated with fasting plasma glucose levels. Science 320, 1085-1088.

Coutinho, M., Gerstein, H. C., Wang, Y., and Yusuf, S. (1999). The relationship between glucose and incident cardiovascular events. A metaregression analysis of published data from 20 studies of 95,783 individuals followed for 12.4 years Diabetes Care 22, 233-240.

Craft, S. (2005). Insulin resistance syndrome and Alzheimer's disease: age- and obesity-related effects on memory, amyloid, and inflammation. Neurobiol. Aging 26, 65-69.

Craik, F. I., and McDowd, J. M. (1987). Age differences in recall and recognition. J. Exp. Psychol. Learn. Mem. Cog. 13, 474-479.

Dempster, E., Toulopoulou, T., McDonald, C., Bramon, E., Walshe, M., Filbey, F., Wickham, H., Sham, P. C., Murray, R. M., and Collier, D. A. (2005). Association between BDNF val66 met genotype and episodic memory. Am. J. Med. Genet B Neuropsychiatr. Genet. 134, 73-75.

Egan, M. F., Kojima, M., Callicott, J. H., Goldberg, T. E., Kolachana, B. S. Bertolino, A., Zaitsev, E., Gold, B., Goldman, D., Dean, M., Lu, B., and Weinberger, D. R. (2003). The BDNF val66met polymorphism affects activitydependent secretion of BDNF and human memory and hippocampal function. Cell 112, 257-269.

Finkbeiner, S. (2000). Calcium regulation of the brain-derived neurotrophic factor gene. Cell Mol. Life Sci. 57, 394-401.

Finkel, D., Pedersen, N., and McGue, M. (1995). Genetic influences on memory performance in adulthood: comparison of Minnesota and Swedish twin data Psychol. Aging 10, 437-446.

Folstein, M. F., Folstein, S. E., and McHugh, P. R. (1975). Mini-mental state A practical method for grading the cognitive state of patients for the clinician. J. Psychiatr. Res. 12, 189-198.

Gold, P. E. (2005). Glucose and age-related changes in memory. Neurobiol. Aging 1 (Suppl), 60-64, Epub 2005 Oct 11.

Goldberg, T. E., Iudicello, J., Russo, C., Elvevåg, B., Straub, R., Egan, M. F., and Weinberger, D. R. (2008). Val66Met polymorphism significantly affects d' in verbal recognition memory at short and long delays. Biol. Psychol. 77, 20-24.

Hansell, N. K., James, M. R., Duffy, D. L., Birley, A. J., Luciano, M., Geffen, G. M., Wright, M. J., Montgomery, G. W., and Martin, N. G. (2007). Effect of the BDNF V166M polymorphism on working memory in healthy adolescents Genes Brain Behav. 6, 260-268.

Hariri, A. R., Goldberg, T. E., Mattay, V. S., Kolachana, B. S., Callicott, J. H., Egan, M. F., and Weinberger, D. R. (2003). Brain-derived neurotrophic factor val66met polymorphism affects human memory-related hippocampal activity and predicts memory performance. J. Neurosci. 23, 6690-6694.

Harris, S. E., Fox, H., Wright, A. F., Hayward, C., Starr, J. M., Whalley, L. J., and Deary, I. J. (2006). The brain-derived neurotrophic factor Val66Met polymorphism is associated with age-related change in reasoning skills. Mol. Psychiatry 11, 505-513.

Ho, B. C., Milev, P., O'Leary, D. S., Librant, A., Andreasen, N. C., and Wassink, T. H. (2006). Cognitive and magnetic resonance imaging brain morphometric correlates of brain-derived neurotrophic factor Val66Met gene polymorphism in patients with schizophrenia and healthy volunteers. Arch. Gen Psychiatry 63, 731-740.

Kaplan, R. J., Greenwood, C. E., Winocur, G., and Wolever, T. M. (2000). Cognitive performance is associated with glucose regulation in healthy elderly person and can be enhanced with glucose and dietary carbohydrates. Am. J. Clin. Nutr. 72, 825-836.

Kaplan, R. J., Greenwood, C. E., Winocur, G., and Wolever, T. M. (2001). Dietary protein, carbohydrate, and fat enhance memory performance in the healthy elderly. Am. J. Clin. Nutr. 74, 687-693.
Krabbe, K. S., Nielsen, A. R., Krogh-Madsen, R., Plomgaard, P., Rasmussen, P., Erikstrup, C., Fischer, C. P., Lindegaard, B., Petersen, A. M., Taudorf, S. Secher, N. H., Pilegaard, H., Bruunsgaard, H., and Pedersen, B. K. (2007). Brain-derived neurotrophic factor (BDNF) and type 2 diabetes. Diabetologia 50, 431-438.

Lynch, G., Rex, C. S., and Gall, C. M. (2006). Synaptic plasticity in early aging. Ageing Res. Rev. 5, 255-280.

Lyons, W. E., Mamounas, L. A., Ricaurte, G. A., Coppola, V., Reid, S. W., Bora, S. H., Wihler, C., Koliatsos, V. E., and Tessarollo, L. (1999). Brain-derived neurotrophic factor-deficient mice develop aggressiveness and hyperphagia in conjunction with brain serotonergic abnormalities. Proc. Natl. Acad. Sci. USA 96, 15239-15244.

Mattson, M. P., Maudsley, S., and Martin, B. (2004). A neural signaling triumvirate that influences ageing and age-related disease: insulin/IGF-1, BDNF and serotonin. Ageing Res. Rev. 3, 445-464.

Messier, C. (2004). Glucose improvement of memory: a review. Eur. J. Pharmacol $490,33-57$.

Miyajima, F., Ollier, W., Mayes, A., Jackson, A., Thacker, N., Rabbitt, P., Pendleton, N., Horan, M., and Payton, A. (2007). Brain-derived neurotrophic factor polymorphism Val66Met influences cognitive abilities in the elderly. Genes Brain Behav. 7, 411-417.

Nakagawa, T., Ono-Kishino, M., Sugaru, E., Yamanaka, M., Taiji, M., and Noguchi, H (2002). Brain-derived neurotrophic factor (BDNF) regulates glucose and energy metabolism in diabetic mice. Diabetes Metab. Res. Rev. 18, 185-191.

Old, S. R., and Naveh-Benjamin, M. (2008). Differential effects of age on item and associative measures of memory: a meta-analysis. Psychol. Aging 23, $104-118$.

Radloff, L. S. (1977). The CES-D scale: a self-report depression scale for research in the general population. Appl. Psychol. Meas. 1, 385-401.

Raz, N., Rodrigue, K. M., Kennedy, K. M., and Land, S. (in press). Genetic and vascular modifiers of age-sensitive cognitive skills: effects of COMT, BDNF, ApoE and hypertension. Neuropsychology.

Riby, L. M., Marriott, A., Bullock, R., Hancock, J., Smallwood, J., and McLaughlin, J. (2008). The effects of glucose ingestion and glucose regulation on memory performance in older adults with mild cognitive impairment. Eur. J. Clin. Nutr. [Epub ahead of print].

Rolandsson, O., Backeström, A., Eriksson, S., Hallmans, G., and Nilsson, L.-G. (2008). Increased glucose levels are associated with episodic memory in nondiabetic women. Diabetes 57, 440-443.

Sohrabji, F., and Lewis, D. K. (2006). Estrogen-BDNF interactions: implications for neurodegenerative diseases. Front. Neuroendocrinol. 27, 404-414.

Stolerman, E. S., Manning, A. K., McAteer, J. B., Dupuis, J., Fox, C. S., Cupples, L. A., Meigs, J. B., and Florez, J. C. (2008). Haplotype structure of the ENPP1 gene and nominal association of the K121Q polymorphism with glycemic traits in the Framingham heart study. Diabetes 57, 1971-1977.

Vanhanen, M., Koivisto, K., Karjalainen, L., Helkala, E. -L., Laakso, M., Soininen, H., and Riekkinen, P. (1997). Risk for non-insulin dependent diabetes in the normoglycaemic elderly is associated with impaired cognitive function. Neuroreport 8, 1527-1530.

Verhaeghen, P., Marcoen, A., and Goossens, L. (1993). Facts and fiction about memory aging: a quantitative integration of research findings. J. Gerontology. 48, P157-P171.

Wechsler, D. (1987). Manual for Wechsler Memory Scale-Revised. San Antonio, Psychological Corporation.

Woodcock, R.W., and Johnson, M. B. (1989).Woodcock-Johnson Psychoeducational Battery - Revised. Allen, DLM Teaching Resources.

Woodcock, R. W., and Mather, N. (1989). Woodcock-Johnson tests of cognitive ability - standard and supplemental batteries: examiner's manual. In Woodcock-Johnson Psychoeducational Battery-Revised, R. W. Woodcock and M. B. Johnson, eds (Itasca, IL: Riverside Publishing), pp. 116-120.

Yaffe, K., Blackwell, T., Kanaya, A. M., Davidowitz, N., Barrett-Connor, E., and Krueger, K. (2004). Diabetes, impaired fasting glucose, and development of cognitive impairment in older women. Neurology 63, 658-663. 